\title{
Matrix operator inequalities based on Mond, Pecaric, Jensen and Ando's
}

\author{
Xue Lanlan and Wu Junliang*
}

\author{
* Correspondence: jlwu678@tom. \\ com \\ College of Mathematics and \\ Statistics of Chongqing University, \\ Chongqing 401331, China
}

\begin{abstract}
This work is concerned with discussing matrix operator's inequalities based on Mond and Pecaric's matrix inequalities and matrix operator inequalities for convex and concave functions that occur in inequalities of Jensen and Ando's for positive linear maps.

Keywords: positive definite, convex (concave) function, operator mean, Hermitian matrix
\end{abstract}

\section{Introduction}

Operator inequalities play important roles in various mathematical and statistical contexts. In early of study operator inequalities, there have been many research results, where useful references centered mainly literatures [1-4]. In nearly 20 years, some new research results of operator inequalities continuously appears, readers may refer to Mond and Pecaric [5], Beesack [1], Hansen [6] and Kubo and Ando [2] and so on. In [5], Mond Pecaric gave several matrix operator inequalities associated with positive linear maps by means of concavity and convexity theorems. Beesack and Pĕcarić in [1] showed the Jensen's inequality for real valued convex functions. Hansen in [6] also gave operator inequalities associated with Jensen's inequality. In this article, we will give some new operator inequalities of matrix version based on Mond, Pecaric, Jensen and Ando's.

This article is organized as follows: In Section 2, based on Mond and Pecaric's operator inequalities we give matrix operator inequalities of real valued convex functions. In Section 3, we first present some new matrix operator inequalities by means of Jensen's and Ando's matrix inequalities, then we give Jensen inequality for convex functions in matrix form and conduct further research on the properties of Jensen inequality.

Throughout the article, $A_{\mathrm{j}}(\mathrm{j}=1,2, \ldots, \mathrm{k})$ denote positive definite Hermitian matrices of order $n \times n$ with eigenvalues in interval $[m, M](0<m<M), U_{j}(j=1,2, \ldots, k)$ denote $r \times n$ matrices such that $\sum_{j=1}^{k} U_{j} U_{j}^{*}=I$. If $A$ is a $n \times n$ Hermitian matrix, then there exists a unitary matrix $U$ such that $A=U^{*}\left[\lambda_{1}, \ldots, \lambda_{n}\right] U$, where $\left[\lambda_{1}, \ldots, \lambda_{n}\right]$ is diagonal matrix and $\lambda_{i}$ $(i=1,2, \ldots, n)$ are eigenvalues of $A$, respectively. $f(A)$ is then defined by $f(A)=U^{*}[f$ $\left.\left(\lambda_{1}\right), \ldots, f\left(\lambda_{n}\right)\right] U$. $A \geq B$ means $A-B$ is positive semi-definite. 
If $F(t)=F(f(t), g(t))$, then we use $F(A)$ to denote matrix operator of $F(f(A), g(A))$, it means that $F(A)=F(f(A), g(A))$, while $F(A, B)$ denotes the matrix function of two variables as it was well defined in some known articles.

\section{Matrix operator inequalities based on Mond and Pecaric's}

In [5], Mond and Pecaric once gave two important matrix inequalities which are the following Theorems $\mathrm{A}$ and $\mathrm{B}$ :

Theorem A. Let $A_{\mathrm{j}}(\mathrm{j}=1,2, \ldots, \mathrm{k})$ be Hermitian matrices of order $n \times n$ with eigenvalues in interval $[m, M]$, and let $U_{\mathrm{j}}(\mathrm{j}=1,2, \ldots, \mathrm{k})$ be $r \times n$ matrices such that $\sum_{j=1}^{k} U_{j} U_{j}^{*}=I$. If $f$ is a real valued continuous convex function on $[m, M]$, then

$$
\sum_{j=1}^{k} U_{j} f\left(A_{j}\right) U_{j}^{*} \leq \frac{M I-\sum_{j=1}^{k} U_{j} A_{j} U^{*}}{M-m} f(m)+\frac{\sum_{j=1}^{k} U_{j} A_{j} U^{*}-m I}{M-m} f(M) .
$$

Theorem B. Let $A_{\mathrm{j}}(\mathrm{j}=1,2, \ldots, \mathrm{k})$ be Hermitian matrices of order $n \times n$ with eigenvalues in interval $[m, M]$, and let $U_{\mathrm{j}}(\mathrm{j}=1,2, \ldots, \mathrm{k})$ be $r \times n$ matrices those such that $\sum_{j=1}^{k} U_{j} U_{j}^{*}=I$. If $f$ is a continuous convex function on $[m, M], J$ is an interval such that $J \supset[m, M]$ and $F(u, v)$ is a real valued continuous function defined on $J \times J$ and it is a matrix increasing function to its first variable, then

$$
\begin{aligned}
F\left(\sum_{j=1}^{k} U_{j} f\right. & \left.\left(A_{j}\right) U_{j}^{*}, f\left(\sum_{j=1}^{k} U_{j} A_{j} U_{j}^{*}\right)\right) \\
& \leq\left\{\max _{x \in[m, M]} F\left[\frac{M-x}{M-m} f(m)+\frac{x-m}{M-m} f(M), f(x)\right]\right\} I \\
& =\left\{\max _{\theta \in[0,1]} F[\theta f(m)+(1-\theta) f(M), f(\theta m+(1-\theta) M)]\right\} I .
\end{aligned}
$$

From Theorems A and B, we can make a more profound study and get more results which have the extensive meaning.

Theorem 2.1. Let $A_{\mathrm{j}}(\mathrm{j}=1,2, \ldots, \mathrm{k})$ be Hermitian matrices of order $n \times n$ with eigenvalues in interval $[m, M]$, and $U_{\mathrm{j}}(\mathrm{j}=1,2, \ldots, \mathrm{k})$ be $r \times n$ matrices such that $\sum_{j=1}^{k} U_{j} U_{j}^{*}=I$. If $f$ is a real valued continuous convex function on $[m, M]$, then

$$
\begin{aligned}
\sum_{j=1}^{k} U_{j} f\left(m I+M I-A_{j}\right) U_{j}^{*} & \leq \frac{\sum_{j=1}^{k} U_{j} A_{j} U_{j}^{*}-m I}{M-m} f(m)+\frac{M I-\sum_{j=1}^{k} U_{j} A_{j} U_{j}^{*}}{M-m} f(M) \\
& \leq f(m) I+f(M) I-\sum_{j=1}^{k} U_{j} A_{j} U_{j}^{*} .
\end{aligned}
$$

Proof. By the proof of Theorem 1 of [5], we know that for any real valued convex function, the following inequality holds 


$$
\begin{aligned}
f(m+M-z) & \leq \frac{M-(m+M-z)}{M-m} f(m)+\frac{(m+M-z)-m}{M-m} f(M) \\
& =\frac{z-m}{M-m} f(m)+\frac{M-z}{M-m} f(M) \\
& =f(m)+f(M)-\left(\frac{M-z}{M-m} f(m)+\frac{z-m}{M-m} f(M)\right) \\
& \leq f(m)+f(M)-f(z) \quad(m+M-z \in[m, M]) .
\end{aligned}
$$

It follows at once from the above inequality that

$$
f\left(m I+M I-A_{j}\right) \leq f(m) I+f(M) I-f\left(A_{j}\right),
$$

where $m I \leq A_{j} \leq M I(\mathrm{j}=1,2, \ldots, \mathrm{k})$. The following matrix inequality can be deduced from above one

$$
\sum_{j=1}^{k} U_{j} f\left(m I+M I-A_{j}\right) U_{j}^{*} \leq f(m) I+f(M) I-\sum_{j=1}^{k} U_{j} f\left(A_{j}\right) U_{j}^{*} .
$$

Similarly, we can get the following theorem:

Theorem 2.2. Let $A_{\mathrm{j}}(\mathrm{j}=1,2, \ldots, \mathrm{k})$ be Hermitian matrices of order $n \times n$ with eigenvalues in the interval $[m, M]$, and let $U_{\mathrm{j}}(\mathrm{j}=1,2, \ldots, \mathrm{k})$ be $r \times n$ matrices such that $\sum_{j=1}^{k} U_{j} U_{j}^{*}=I$. If $f$ is a real valued continuous convex function on $[m, M]$, then

$$
\begin{aligned}
\sum_{j=1}^{k} U_{j} f\left(m I+M I-A_{j}\right) U_{j}^{*} & \geq f\left(\frac{m+M}{2}\right) I-\sum_{j=1}^{k} U_{j} f\left(A_{j}\right) U_{j}^{*} \\
& \geq 2 f\left(\frac{m+M}{2}\right) I-\left(\frac{M I-\sum_{j=1}^{k} U_{j} A_{j} U_{j}^{*}}{M-m} f(m)+\frac{\sum_{j=1}^{k} U_{j} A_{j} U_{j}^{*}-m I}{M-m} f(M)\right) .
\end{aligned}
$$

Proof. By the proof of Theorem 2.1, we know that for any a real valued convex function, the following inequality holds:

$$
\begin{aligned}
f(m+M-z) & \geq 2 f\left(\frac{m+M}{2}\right)-f(z) \\
& \geq 2 f\left(\frac{m+M}{2}\right)-\left(\frac{M-z}{M-m} f(m)+\frac{z-m}{M-m} f(M)\right) \quad(m+M-z \in[m, M]) .
\end{aligned}
$$

It follows from the above inequality that

$$
\begin{aligned}
f\left(m I+M I-A_{j}\right) & \geq f\left(\frac{m+M}{2}\right) I-f\left(A_{j}\right) \\
& \geq 2 f\left(\frac{m+M}{2}\right) I-\left(\frac{M I-A_{j}}{M-m} f(m)+\frac{A_{j}-m I}{M-m} f(M)\right),
\end{aligned}
$$

where $m I \leq A_{j} \leq M I(\mathrm{j}=1,2, \ldots, \mathrm{k})$. Further, the following matrix inequality can be deduced from above one 


$$
\begin{aligned}
\sum_{j=1}^{k} U_{j} f\left(m I+M I-A_{j}\right) U_{j}^{*} & \geq f\left(\frac{m+M}{2}\right) I-\sum_{j=1}^{k} U_{j} f\left(A_{j}\right) U_{j}^{*} \\
& \geq 2 f\left(\frac{m+M}{2}\right) I-\left(\frac{M I-\sum_{j=1}^{k} U_{j} A_{j} U_{j}^{*}}{M-m} f(m)+\frac{\sum_{j=1}^{k} U_{j} A_{j} U_{j}^{*}-m I}{M-m} f(M)\right) .
\end{aligned}
$$

As a special case of Theorem $\mathrm{B}$, we give the following:

Theorem 2.3. Let $A_{\mathrm{j}}(\mathrm{j}=1,2, \ldots, \mathrm{k})$ be Hermitian matrices of order $n \times n$ with eigenvalues in interval $[m, M]$ and $U_{\mathrm{j}}(\mathrm{j}=1,2, \ldots, \mathrm{k})$ be $r \times n$ matrices such that $\sum_{j=1}^{k} U_{j} U_{j}^{*}=I$. If $f$ is a continuous convex function on $[m, M], J$ is an interval which satisfy $J \supset[m, M], F(u, v)=u-v$ is matrix increasing in its first variable, then the following inequality holds:

$$
\sum_{j=1}^{k} U_{j} f\left(A_{j}\right) U_{j}^{*}-f\left(\sum_{j=1}^{k} U_{j} A_{j} U_{j}^{*}\right) \leq \max _{\theta \in[0,1]}[\theta f(m)+(1-\theta) f(M)-f(\theta m+(1-\theta) M)] I .
$$

We can also get $\sum_{j=1}^{k} U_{j} f\left(A_{j}\right) U_{j}^{*} \leq \max _{\theta \in[0,1]}\left[\frac{\theta f(m)+(1-\theta) f(M)}{f(\theta m+(1-\theta) M)}\right] f\left(\sum_{j=1}^{k} U_{j} A_{j} U_{j}^{*}\right)$.

\section{Matrix operator inequalities based on Jensen and Ando's}

In this section, we discuss two issues, the first is matrix operator inequalities that get based on an important lemma, and the second is the Jensen inequality of matrix form and further research on the properties of the inequality.

In the first part, we suppose $A$ is a positive definite Hermitian matrix of order $n \times n$ with $m I \leq A \leq M I, f(t)$ is defined as a nonnegative concave function on $[m, M]$ with 0 $<m<M$, then $f(A)$ is defined as a positive operator by the usual functional calculus. A real valued function $f$ is said to be operator monotone on interval $[m, M]$ if $A \geq B \Rightarrow f$ $(A) \geq f(B)$ for every positive definite Hermitian matrices $A$ and $B$ whose eigenvalues are contained on $[m, M]$, and $f$ is said to be operator concave on $[m, M]$ if $f(\lambda A+(1-\lambda) B)$ $\geq \lambda f(A)+(1-\lambda) f(B)$ for all real numbers $0 \leq \lambda \leq 1$ and every positive definite Hermitian matrices $A$ and $B$ whose eigenvalues are contained in $[m, M]$.

We begin with the following lemma.

Lemma 3.1. Let $A_{\mathrm{j}}(\mathrm{j}=1,2, \ldots, \mathrm{k})$ be positive definite Hermitian matrices of order $n \times n$ with eigenvalues in $[m, M], f(t)$ be a nonnegative real value continuous strictly concave twice differentiable function on $[m, M]$ with $0<m<M$, and $U_{\mathrm{j}}(\mathrm{j}=1,2, \ldots, \mathrm{k})$ be $r \times n$ matrices such that $\sum_{j=1}^{k} U_{j} U_{j}^{*}=I$. Then for any given $\alpha>0$,

$$
\begin{gathered}
\sum_{j=1}^{k} U_{j} f\left(A_{j}\right) U_{j}^{*} \geq \alpha f\left(\sum_{j=1}^{k} U_{j} A_{j} U_{j}^{*}\right)+\beta I \text { holds for } \\
\beta=\beta(m, M, f, \alpha)=a t+b-\alpha f\left(t_{0}\right),
\end{gathered}
$$


where $t_{0}$ is defined as the unique solution of $f^{\prime}(t)=\frac{a}{\alpha} \quad$ when $f^{\prime}(M) \leq \frac{a}{\alpha} \leq f^{\prime}(m)\left(a=\frac{f(M)-f(m)}{M-m}, b=\frac{M f(m)-m f(M)}{M-m}\right) ; \quad$ Otherwise $\quad t_{0} \quad$ is defined as $M$ or $m$ according as $\frac{a}{\alpha} \leq f^{\prime}(M)$ or $f^{\prime}(m) \leq \frac{a}{\alpha}$.

Proof. We take $h(t)=a t+b-\alpha f\left(t_{0}\right)$. Since $f(t)$ is strictly concave, its derivative $f(t)$ is strictly decreasing. Hence, if $f^{\prime}(M) \leq \frac{a}{\alpha} \leq f^{\prime}(m)$, then $h^{\prime}(t)=0$ occurs in interval $[m$, $M]$ only at point $t_{0}$. Since $h^{\prime \prime}(t)=-\alpha f^{\prime \prime}(t)>0$, this means that $\min _{m \leq t \leq M} h(t)=h\left(t_{0}\right) \equiv \beta$. Next, we consider $\frac{a}{\alpha} \leq f^{\prime}(M)$. Since, $h^{\prime}(t)<0, h(t)$ is decreasing on $[m, M]$. Therefore, we can deduce that $\min _{m \leq t \leq M} h(t)=h\left(t_{0}\right) \equiv \beta$ as $t_{0}=M$. Similarly, if $f^{\prime}(m) \leq \frac{a}{\alpha}$, then we deduce that $\min _{m \leq t \leq M} h(t)=h\left(t_{0}\right) \equiv \beta$ as $t_{0}=m$. It follows that $a t+b \geq \alpha f(t)+\beta$ for $t \in$ $[m, M]$.

If applying the inequality above to $\sum_{j=1}^{k} U_{j} A_{j} U_{j}^{*}$, we have

$$
a \sum_{j=1}^{k} U_{j} A_{j} U_{j}^{*}+b I \geq \alpha f\left(\sum_{j=1}^{k} U_{j} A_{j} U_{j}^{*}\right)+\beta I,
$$

where $m I \leq \sum_{j=1}^{k} U_{j} A_{j} U_{j}^{*} \leq M I$.

On the other hand, since $f(t)$ is concave, we have $f(t) \geq a t+b$ for $t \in[m, M]$, applying this inequality to $A_{j}$, we have $f\left(A_{j}\right) \geq a A_{j}+b I$, and then

$$
\sum_{j=1}^{k} U_{j} f\left(A_{j}\right) U_{j}^{*} \geq a \sum_{j=1}^{k} U_{j} A_{j} U_{j}^{*}+b I .
$$

Combining these two inequalities we obtain

$$
\sum_{j=1}^{k} U_{j} f\left(A_{j}\right) U_{j}^{*} \geq \alpha f\left(\sum_{j=1}^{k} U_{j} A_{j} U_{j}^{*}\right)+\beta I .
$$

凶

Use a similar method, the following Theorem can be proved easily.

Remark 3.1. If we put $\alpha=1$ in Lemma 3.1, then

$$
-\beta I \geq f\left(\sum_{j=1}^{k} U_{j} A_{j} U_{j}^{*}\right)-\sum_{j=1}^{k} U_{j} f\left(A_{j}\right) U_{j}^{*}
$$

holds for $\beta=a t+b-f\left(t_{0}\right)$ and $t_{0}$ satisfy $f^{\prime}(t)=a$. Since $f$ is strictly concave, it can deduce that $f^{\prime}(M) \leq a \leq f^{\prime}(m)$.

The following corollaries are the special case of Lemma 3.1. 
Corollary 3.1. Let, $A_{\mathrm{j}}(\mathrm{j}=1,2, \ldots, \mathrm{k})$ be positive definite Hermitian matrices of order $n$ $\times n$ with eigenvalues in interval $[m, M]$ that $0<m I \leq A_{j} \leq M I$ and $0<p<1$

(resp. $p<0, p>1$ ), and let $U_{\mathrm{j}}(\mathrm{j}=1,2, \ldots, \mathrm{k})$ be $r \times n$ matrices such that $\sum_{j=1}^{k} U_{j} U_{j}^{*}=I$.

Then for any given $\alpha>0$

$$
\sum_{j=1}^{k} U_{j} A_{j}^{p} U_{j}^{*} \geq \alpha\left(\sum_{j=1}^{k} U_{j} A_{j} U_{j}^{*}\right)^{p}+\beta I\left(\operatorname{resp} \cdot \sum_{j=1}^{k} U_{j} A_{j}^{p} U_{j}^{*} \leq \alpha\left(\sum_{j=1}^{k} U_{j} A_{j} U_{j}^{*}\right)^{p}+\beta I\right)
$$

holds for

$$
\beta=\beta\left(m, M, t^{p}, \alpha\right)=\left\{\begin{array}{c}
\alpha(p-1)\left(\frac{1}{\alpha p} \frac{M^{p}-m^{p}}{M-m}\right)^{\frac{p}{p-1}}+\frac{M m^{p}-m M^{p}}{M-m} \\
\quad \operatorname{if} p M^{p-1} \leq \frac{1}{\alpha} \frac{M^{p}-m^{p}}{M-m} \leq p m^{p-1}, \\
\min \left\{(1-\alpha) M^{p},(1-\alpha) m^{p}\right\} \text { otherwise, }
\end{array}\right.
$$

resp.

$$
\beta=\beta\left(m, M, t^{p}, \alpha\right)=\left\{\begin{array}{c}
\alpha(p-1)\left(\frac{1}{\alpha p} \frac{M^{p}-m^{p}}{M-m}\right)^{\frac{p}{p-1}}+\frac{M m^{p}-m M^{p}}{M-m} \\
\quad \text { if } p m^{p-1} \leq \frac{1}{\alpha} \frac{M^{p}-m^{p}}{M-m} \leq p M^{p-1}, \\
\max \left\{(1-\alpha) M^{p},(1-\alpha) m^{p}\right\} \text { otherwise. }
\end{array}\right.
$$

Proof. We only prove the former, as in proof of Theorem 3.1, let $f(t)=t^{p}$, since $t_{0}$ is defined as the unique solution of $f^{\prime}(t)=\frac{a}{\alpha}$ when $f^{\prime}(m) \leq \frac{a}{\alpha} \leq f^{\prime}(M)$, so we get $t_{0}=\left(\frac{a}{p \alpha}\right)^{\frac{1}{p-1}} \quad$ and $\quad \beta=\alpha(p-1)\left(\frac{1}{\alpha p} \frac{M^{p}-m^{p}}{M-m}\right)^{\frac{p}{p-1}}+\frac{M m^{p}-m M^{p}}{M-m} \quad$ by $f^{\prime}\left(t_{0}\right)=\frac{a}{\alpha}=p t^{p-1}$, when $p M^{p-1} \leq \frac{1}{\alpha} \frac{M^{p}-m^{p}}{M-m} \leq p m^{p-1}$, where $a=\frac{f(M)-f(m)}{M-m}$ and $b=\frac{M f(m)-m f(M)}{M-m}$. Otherwise $\beta=\min \left\{(1-\alpha) M^{p},(1-\alpha) m^{p}\right\}$. Hence the results obtained by the conclusion of Lemma 3.1 since $f(t)=t^{p}$.

As a special case of Corollary3.1, we get the following corollary.

Corollary 3.2. Let $A_{\mathrm{j}}(\mathrm{j}=1,2, \ldots, \mathrm{k})$ be positive definite Hermitian matrices of order $n \times$ $n$ with eigenvalues in the interval $[m, M]$ that $0<m I \leq A_{j} \leq M I$, let $U_{\mathrm{j}}(\mathrm{j}=1,2, \ldots, \mathrm{k})$ be $r \times$ $n$ matrices such that $\sum_{j=1}^{k} U_{j} U_{j}^{*}=I$. Then for any given $\alpha>0$

$$
\sum_{j=1}^{k} U_{j} A_{j}^{-1} U_{j}^{*} \leq \alpha\left(\sum_{j=1}^{k} U_{j} A_{j} U_{j}^{*}\right)^{-1}+\beta I
$$


holds for

$$
\beta=\beta\left(m, M, t^{-1}, \alpha\right)=\left\{\begin{array}{cc}
\frac{M+m}{m M}-2 \sqrt{\frac{\alpha}{M m}} & \text { if } \frac{m}{M} \leq \alpha \leq \frac{M}{m}, \\
\max \left\{\frac{1-\alpha}{m}, \frac{1-a}{M}\right\} & \text { if either } 0<\alpha<\frac{m}{M} \text { or } \frac{M}{m}<\alpha .
\end{array}\right.
$$

In particular,

$$
\begin{gathered}
\sum_{j=1}^{k} U_{j} A_{j}^{-1} U_{j}^{*}-\left(\sum_{j=1}^{k} U_{j} A_{j} U_{j}^{*}\right)^{-1} \leq \frac{(\sqrt{M}-\sqrt{m})^{2}}{M m} I . \\
\sum_{j=1}^{k} U_{j} A_{j}^{-1} U_{j}^{*} \leq \frac{(M+m)^{2}}{4 M m}\left(\sum_{j=1}^{k} U_{j} A_{j} U_{j}^{*}\right)^{-1} .
\end{gathered}
$$

Proof. The results obtain by the conclusion of Corollary 3.1 as $f(t)=t^{-1}$.

Remark 3.2. If we put $\alpha=1$ and $p=2$ in Corollary 3.1, then

$$
\sum_{j=1}^{k} U_{j} A_{j}^{2} U_{j}^{*}-\left(\sum_{j=1}^{k} U_{j} A_{j} U_{j}^{*}\right)^{2} \leq \frac{(M-m)^{2}}{4} I .
$$

The results were obtained by Liu and Neudecker [7].

The theory of operator means for positive linear operators on a Hilbert space which in connection with Löwner's theory for operator monotone functions was established by Kubo and Ando [8]. In the following discussion, as matrix versions, we will give the generalization of matrix inequalities through the theory of operator means which we apply it to derive Jensen's and Ando's matrix inequalities. For that we give the following definition:

A map $(A, B) \rightarrow A \sigma B$ is called an operator mean if the following conditions are satisfied:

(M1) Monotonicity: $A \leq C$ and $B \leq D$ imply $A \sigma B \leq C \sigma D$;

(M2) upper continuity: $A_{n} \downarrow A$ and $B_{n} \downarrow B$ imply $A_{n} \sigma B_{n} \downarrow A \sigma B$;

(M3) transformer inequality: $U(A \sigma B) U^{*} \leq\left(U A U^{*}\right) \sigma\left(U B U^{*}\right)$ for any operator $U$.

If $f$ is nonnegative operator concave function, we define an operator mean $\sigma$ through the formula

$$
A \sigma B=A^{\frac{1}{2}} f\left(A^{-\frac{1}{2}} B A^{-\frac{1}{2}}\right) A^{\frac{1}{2}}
$$

for all positive definite Hermitian matrices $A$ and $B$ of order $n \times n$. Simple example of operator means is the geometric mean \# defined by

$$
A \#_{\frac{1}{2}} B=A^{\frac{1}{2}}\left(A^{-\frac{1}{2}} B A^{-\frac{1}{2}}\right)^{\frac{1}{2}} A^{\frac{1}{2}} .
$$


Before proceeding further, we present a useful lemma.

Lemma 3.2. Let $f$ be a nonnegative operator concave function on $[m, M]$ with $0<m$ $<M$, for all positive definite Hermitian matrices $A$ and $B$, let $U$ be any $r \times n$ matrix such that $U U^{*}=I$. Then the following statements are mutually equivalent:

(i) $U(A \sigma B) U^{*} \leq\left(U A U^{*}\right) \sigma\left(U B U^{*}\right)$,

(ii) $U f(A) U^{*} \leq f\left(U A U^{*}\right)$.

Proof. By (i), (ii) is obvious as $f$ is concave. Then we will show that (ii) implies (i). In fact,

$$
\begin{aligned}
U(A \sigma B) U^{*} & =U A^{\frac{1}{2}} f\left(A^{-\frac{1}{2}} B A^{-\frac{1}{2}}\right) A^{\frac{1}{2}} U^{*} \\
& =\left(U A U^{*}\right)^{\frac{1}{2}}\left(U A U^{*}\right)^{-\frac{1}{2}} U A^{\frac{1}{2}} f\left(A^{-\frac{1}{2}} B A^{-\frac{1}{2}}\right) A^{\frac{1}{2}} U^{*}\left(U A U^{*}\right)^{-\frac{1}{2}}\left(U A U^{*}\right)^{\frac{1}{2}} \\
& =\left(U A U^{*}\right)^{\frac{1}{2}}\left[\left(\left(U A U^{*}\right)^{-\frac{1}{2}} U A^{\frac{1}{2}}\right) f\left(A^{-\frac{1}{2}} B A^{-\frac{1}{2}}\right)\left(A^{\frac{1}{2}} U^{*}\left(U A U^{*}\right)^{-\frac{1}{2}}\right)\right]\left(U A U^{*}\right)^{\frac{1}{2}} \\
& \leq\left(U A U^{*}\right)^{\frac{1}{2}}\left[f\left(\left(U A U^{*}\right)^{-\frac{1}{2}} U A^{\frac{1}{2}} A^{-\frac{1}{2}} B A^{-\frac{1}{2}} A^{\frac{1}{2}} U^{*}\left(U A U^{*}\right)^{-\frac{1}{2}}\right)\right]\left(U A U^{*}\right)^{\frac{1}{2}} \\
& =\left(U A U^{*}\right)^{\frac{1}{2}} f\left(\left(U A U^{*}\right)^{-\frac{1}{2}} U B U^{*}\left(U A U^{*}\right)^{-\frac{1}{2}}\right)\left(U A U^{*}\right)^{\frac{1}{2}} \\
& =\left(U A U^{*}\right) \sigma\left(U B U^{*}\right)
\end{aligned}
$$

holds for $V V^{*}=\left(U A U^{*}\right)^{-\frac{1}{2}} U A^{\frac{1}{2}} A^{\frac{1}{2}} U^{*}\left(U A U^{*}\right)^{-\frac{1}{2}}=I^{\text {, where }} V=\left(U A U^{*}\right)^{-\frac{1}{2}} U A^{\frac{1}{2}}$.

We now introduce some new theorems of operator means for concave matrix functions.

Theorem 3.1. Let $A$ and $B$ be positive definite Hermitian matrices such that $0<m_{1} I$ $\leq A \leq M_{1} I, 0<m_{2} I \leq B \leq M_{2} I$ and $f(t)$ be a nonnegative real valued continuous twice differentiable function on $[m, M]$ with $0<m<M$, let $U$ be $r \times n$ matrix such that $U U^{*}$ $=I$. Then $U(A \sigma B) U^{*} \geq \alpha\left(U A U^{*}\right) \sigma\left(U B U^{*}\right)+\beta U A U^{*}$ holds for any given $\alpha>0$ and $\beta=\beta$ $(m, M, f, \alpha)=a t+b-\alpha f\left(t_{0}\right)$ where $t_{0}$ is defined as the unique solution of $f^{\prime}(t)=\frac{a}{\alpha}$

when $f^{\prime}(M) \leq \frac{a}{\alpha} \leq f^{\prime}(m)$, here $a=\frac{f(M)-f(m)}{M-m}$ and $b=\frac{M f(m)-m f(M)}{M-m}$;

Otherwise $t_{0}$ is defined as $M$ or $m$ according as $\frac{a}{\alpha} \leq f^{\prime}(M)$ or $f^{\prime}(m) \leq \frac{a}{\alpha}$, where $m=\frac{m_{2}}{M_{1}}$ and $M=\frac{M_{2}}{m_{1}}$.

$U(B \sigma A) U^{*} \geq \alpha\left(U B U^{*}\right) \sigma\left(U A U^{*}\right)+\beta U B U^{*}$ holds for which is defined just as above with $m=\frac{m_{1}}{M_{2}}, M=\frac{M_{1}}{m_{2}}$.

Proof. We only prove the former. It follows from Lemma 3.1 that for a given $\alpha>0$

$$
\begin{aligned}
\left(\left(U A U^{*}\right)^{-\frac{1}{2}} U A^{\frac{1}{2}}\right) & f\left(A^{-\frac{1}{2}} B A^{-\frac{1}{2}}\right)\left(A^{\frac{1}{2}} U^{*}\left(U A U^{*}\right)^{-\frac{1}{2}}\right) \\
& \geq \alpha f\left(\left(U A U^{*}\right)^{-\frac{1}{2}} U A^{\frac{1}{2}} A^{-\frac{1}{2}} B A^{-\frac{1}{2}} A^{\frac{1}{2}} U^{*}\left(U A U^{*}\right)^{-\frac{1}{2}}\right)+\beta I
\end{aligned}
$$




$$
\begin{aligned}
& \text { holds for } V V^{*}=\left(U A U^{*}\right)^{-\frac{1}{2}} U A^{\frac{1}{2}} A^{\frac{1}{2}} U^{*}\left(U A U^{*}\right)^{-\frac{1}{2}}=I \text { and } \beta=\beta\left(\frac{m_{2}}{M_{1}}, \frac{M_{2}}{m_{1}}, f, \alpha\right) \text {, } \\
& \text { where } V=\left(U A U^{*}\right)^{-\frac{1}{2}} U A^{\frac{1}{2}} \text { and } \\
& \qquad \begin{aligned}
U(A \sigma B) U^{*} & =U A \frac{1}{2} f\left(A^{-\frac{1}{2}} B A^{-\frac{1}{2}}\right) A^{\frac{1}{2}} U^{*} \\
& =\left(U A U^{*}\right)^{\frac{1}{2}}\left(U A U^{*}\right)^{-\frac{1}{2}} U A^{\frac{1}{2}} f\left(A^{-\frac{1}{2}} B A^{-\frac{1}{2}}\right) A^{\frac{1}{2}} U^{*}\left(U A U^{*}\right)^{-\frac{1}{2}}\left(U A U^{*}\right)^{\frac{1}{2}} \\
& =\left(U A U^{*}\right)^{\frac{1}{2}}\left[\left(\left(U A U^{*}\right)^{-\frac{1}{2}} U A^{\frac{1}{2}}\right) f\left(A^{-\frac{1}{2}} B A^{-\frac{1}{2}}\right)\left(A^{\frac{1}{2}} U^{*}\left(U A U^{*}\right)^{-\frac{1}{2}}\right)\right]\left(U A U^{*}\right)^{\frac{1}{2}} \\
& \leq\left(U A U^{*}\right)^{\frac{1}{2}}\left[\alpha f\left(\left(U A U^{*}\right)^{-\frac{1}{2}} U A^{\frac{1}{2}} A^{-\frac{1}{2}} B A^{-\frac{1}{2}} A^{\frac{1}{2}} U^{*}\left(U A U^{*}\right)^{-\frac{1}{2}}\right)+\beta I\right]\left(U A U^{*}\right)^{\frac{1}{2}} \\
& =\left(U A U^{*}\right)^{\frac{1}{2}}\left[\alpha f\left(\left(U A U^{*}\right)^{-\frac{1}{2}} U B U^{*}\left(U A U^{*}\right)^{-\frac{1}{2}}\right)+\beta I\right]\left(U A U^{*}\right)^{\frac{1}{2}} \\
& =\alpha\left(U A U^{*}\right) \sigma\left(U B U^{*}\right)+\beta U A U^{*} .
\end{aligned}
\end{aligned}
$$

Remark 3.3. If we put $\alpha=1$ in Theorem 3.1, then we have the following:

$$
\begin{aligned}
& \quad-\beta U A U^{*} \geq\left(U A U^{*}\right) \sigma\left(U B U^{*}\right)-U(A \sigma B) U^{*} \\
& \text { resp. }\left(-\beta U B U^{*} \geq\left(U B U^{*}\right) \sigma\left(U A U^{*}\right)-U(B \sigma A) U^{*}\right)
\end{aligned}
$$

holds for $\beta=a t+b-f\left(t_{0}\right)$ and $t_{0}$ such that $f(t)=a$, where $m=\frac{m_{2}}{M_{1}}$ and $M=\frac{M_{2}}{m_{1}}$

$$
\left(\operatorname{resp} . m=\frac{m_{1}}{M_{2}}, M=\frac{M_{1}}{m_{2}}\right)
$$

If we put $f(t)=t \frac{1}{2}$ in Theorem 3.1, the following Corollary 3.3 follows from Theorem 3.1.

Corollary 3.3. Let $A$ and $B$ be positive definite Hermitian matrices such that $0<m_{1} I$ $\leq A \leq M_{1} I$ and $0<m_{2} I \leq B \leq M_{2} I$. Let $U$ be $r \times n$ matrix such that $U U^{*}=I$. Then for any given $\alpha>0$

$$
U\left(A A_{\frac{1}{2}} B\right) U^{*} \geq \alpha\left(U A U^{*}\right) \#_{\frac{1}{2}}\left(U B U^{*}\right)+\beta U A U^{*}
$$

holds for

$$
\beta=\beta\left(m, M, t^{-1}, \alpha\right)=\left\{\begin{array}{l}
\frac{M m^{\frac{1}{2}}-m M^{\frac{1}{2}}}{M-m}-\frac{\alpha^{2}}{4}(\sqrt{M}+\sqrt{m}) \\
\min \left\{(1-\alpha) M^{\frac{1}{2}},(1-\alpha) m^{\frac{1}{2}}\right\}
\end{array} \begin{array}{c}
2 \sqrt{m} \\
\sqrt{M}+\sqrt{m} \\
\text { otherwise }
\end{array}\right.
$$


where $m=\frac{m_{2}}{M_{1}}$ and $M=\frac{M_{2}}{m_{1}}$, and

$$
U\left(B_{\frac{1}{2}} A\right) U^{*} \geq \alpha\left(U B U^{*}\right)_{\frac{1}{2}}\left(U A U^{*}\right)+\beta U B U^{*}
$$

holds for $\beta$ which is defined just as above with $m=\frac{m_{1}}{M_{2}}, M=\frac{M_{1}}{m_{2}}$.

In the following part, according to the traditional Jensen inequality of convex function, we discuss Jensen inequality in matrix form that based on Hermitian matrix and conduct further research on the properties of Jensen inequality.

An inequality be said to be a Jensen inequality of Hermitian matrix is means that a convex function $f$ defined on $I=[m, M]$ satisfies $f\left(\sum_{i=1}^{k} P_{i} A_{i}\right) \leq \sum_{i=1}^{k} P_{i} f\left(A_{i}\right)$, where $A_{i}$ $(i=1, \ldots, k)$ are $n \times n$ Hermitian and commutative matrices with eigenvalues in $[m, M]$, $P_{i}>0(i=1, \ldots, k)$ with $\sum_{i=1}^{k} P_{i}=1$.

Lemma 3.3. Let $f$ be convex function on $I=[m, M], \lambda_{i}(i=1, \ldots, k) \in[m, M]$ and $P_{i}>0$ $(i=1, \ldots, k)$ with $\sum_{i=1}^{k} P_{i}=1, \lambda_{i_{j}}(j=1, \cdots, n) \in[m, M]$, then

$$
\sum_{i=1}^{k} P_{i} f\left(\lambda_{i_{j}}\right)-f\left(\sum_{i=1}^{k} P_{i} \lambda_{i_{j}}\right) \leq \alpha_{j} f(m)+\beta_{j} f(M)-f\left(\alpha_{j} m+\beta_{j} M\right) .
$$

Proof. Since $\lambda_{i_{j}}(j=1, \cdots, n) \in[m, M]$, there are sequences $\left\{u_{i_{j}}\right\},\left\{v_{i_{j}}\right\} \in[0,1]$ with $u_{i_{j}}+v_{i_{j}}=1$, such that $\lambda_{i_{j}}=u_{i_{j}} m+v_{i_{j}} M, j=1,2, \ldots, n$, hence

$$
\begin{aligned}
& \sum_{i=1}^{k} P_{i} f\left(\lambda_{i_{j}}\right)-f\left(\sum_{i=1}^{k} P_{i} \lambda_{i_{j}}\right) \\
& \quad=\sum_{i=1}^{k} P_{i} f\left(u_{i_{j}} m+v_{i_{j}} M\right)-f\left[\sum_{i=1}^{k} P_{i}\left(u_{i_{j}} m+v_{i_{j}} M\right)\right] \\
& \quad=\sum_{i=1}^{k} P_{i} f\left(u_{i j} m+\left(1-u_{i_{j}}\right) M\right)-f\left[\sum_{i=1}^{k} P_{i}\left(u_{i_{j}} m+\left(1-u_{i_{j}}\right) M\right)\right] \\
& \quad \leq \sum_{i=1}^{k} P_{i}\left(u_{i j} f(m)+\left(1-u_{i_{j}}\right) f(M)\right)-f\left[m \sum_{i=1}^{k} P_{i} u_{i_{j}}+M \sum_{i=1}^{k} P_{i}\left(1-u_{i_{j}}\right)\right] \\
& \quad=f(m)\left(\sum_{i=1}^{k} P_{i} u_{i j}\right)+f(M)\left(1-\sum_{i=1}^{k} P_{i} u_{i_{j}}\right)-f\left[m \sum_{i=1}^{k} P_{i} u_{i_{j}}+M\left(1-\sum_{i=1}^{k} P_{i} u_{i_{j}}\right)\right] .
\end{aligned}
$$

Denoting $\sum_{i=1}^{k} P_{i} u_{i_{j}}=\alpha_{j}, 1-\sum_{i=1}^{k} P_{i} u_{i_{j}}=\beta_{j}$, we have that $0 \leq \alpha_{j}, \beta_{j} \leq 1$ with $\alpha_{j}+\beta_{j}=1$.

Consequently,

$$
\sum_{i=1}^{k} P_{i} f\left(\lambda_{i_{j}}\right)-f\left(\sum_{i=1}^{k} P_{i} \lambda_{i_{j}}\right) \leq \alpha_{j} f(m)+\beta_{j} f(M)-f\left(\alpha_{j} m+\beta_{j} M\right) .
$$


From this lemma, we have

Theorem 3.2. Let $f$ be convex function on $I=[m, M], A_{i}(i=1, \ldots, k)$, are Hermitian matrices of order $n \times n$ with eigenvalues in $[m, M]$ and commutative matrices; $P_{i}>0(i$ $=1, \ldots, k)$ with $\sum_{i=1}^{k} P_{i}=1$, then

$$
\sum_{i=1}^{k} P_{i} f\left(A_{i}\right)-f\left(\sum_{i=1}^{k} P_{i} A_{i}\right) \leq \max _{\alpha_{j}}\left[\alpha_{j} f(m)+\beta_{j} f(M)-f\left(\alpha_{j} m+\beta_{j} M\right)\right] I .
$$

Proof. As the proof in the definition, by Lemma 3, we get

$$
\begin{aligned}
& \sum_{i=1}^{k} P_{i} f\left(A_{i}\right)-f\left(\sum_{i=1}^{k} P_{i} A_{i}\right) \\
& \quad=\sum_{i=1}^{k} P_{i} U^{*} \operatorname{diag}\left[f\left(\lambda_{i_{1}}\right), \ldots, f\left(\lambda_{i_{n}}\right)\right] U-f\left[\sum_{i=1}^{k} P_{i} U^{*} \operatorname{diag}\left(\lambda_{i_{1}}, \ldots, \lambda_{i_{n}}\right) U\right] \\
& \quad=U^{*} \operatorname{diag}\left[\sum_{i=1}^{k} P_{i} f\left(\lambda_{i_{1}}\right), \ldots, \sum_{i=1}^{k} P_{i} f\left(\lambda_{i_{n}}\right)\right] U-U^{*} \operatorname{diag}\left[f\left(\sum_{i=1}^{k} P_{i} \lambda_{i_{1}}\right), \ldots, f\left(\sum_{i=1}^{k} P_{i} \lambda_{i_{n}}\right)\right] U \\
& \quad=U^{*} \operatorname{diag}\left[\sum_{i=1}^{k} P_{i} f\left(\lambda_{i_{1}}\right)-f\left(\sum_{i=1}^{k} P_{i} \lambda_{i_{1}}\right), \ldots, \sum_{i=1}^{k} P_{i} f\left(\lambda_{i_{n}}\right)-f\left(\sum_{i=1}^{k} P_{i} \lambda_{i_{n}}\right)\right] U \\
& \quad \leq U^{*} \operatorname{diag}\left[\left(\alpha_{1} f(m)+\beta_{1} f(M)-f\left(\alpha_{1} m+\beta_{1} M\right)\right), \ldots,\left(\alpha_{n} f(m)+\beta_{n} f(M)-f\left(\alpha_{n} m+\beta_{n} M\right)\right)\right] U \\
& \quad \leq \max _{\alpha_{j}}\left[\alpha_{j} f(m)+\beta_{j} f(M)-f\left(\alpha_{j} m+\beta_{j} M\right)\right] I .
\end{aligned}
$$

Apply Theorem 3.2 with $f=x^{2}$, we obtain the following corollary which we call it pre-Gruss inequality of Hermitan matrix.

Corollary 3.4. Let $A_{i}(i=1, \ldots, k), P_{i}$ be defined as above, then

$$
\sum_{i=1}^{k} P_{i} A_{i}{ }^{2}-\left(\sum_{i=1}^{k} P_{i} A_{i}\right)^{2} \leq \frac{1}{4}(M-m)^{2} I .
$$

Proof. By Theorem 3.2, we obtain at once the following inequality

$$
\begin{aligned}
\sum_{i=1}^{k} P_{i} A_{i}^{2}-\left(\sum_{i=1}^{k} P_{i} A_{i}\right)^{2} \leq & \max _{\alpha_{j}}\left(\alpha_{j} m^{2}+\beta_{j} M^{2}-\left(\alpha_{j} m+\beta_{j} M\right)^{2}\right) I \\
& \leq \max _{\alpha_{j}}\left(\alpha_{j} \beta_{j}(M-m)^{2}\right) I \\
& =\frac{1}{4}(M-m)^{2} I .
\end{aligned}
$$

For

$$
P_{1}=P_{2}=\cdots=P_{k}=\frac{1}{k}
$$

Let us define

$$
P_{0}=\left\{\frac{1}{k}, \frac{2}{k}, \cdots, \frac{k-1}{k}\right\} .
$$

From Theorem 3.2, we have 
Corollary 3.5. Let $f, A_{i}(i=1, \ldots, k), P_{i}$ be defined as above, then

$$
\begin{aligned}
& \frac{f\left(A_{1}\right)+f\left(A_{2}\right)+\cdots+f\left(A_{k}\right)}{k}-f\left(\frac{A_{1}+A_{2}+\cdots+A_{k}}{k}\right) \\
& \leq \max _{p \in P_{0}}[p f(m)+(1-p) f(M)-f(p m+(1-p) M)] I .
\end{aligned}
$$

Theorem 3.3. Let $f, A_{i}(i=1, \ldots, k), P_{i}$ be defined as above, then we have

$$
\sum_{i=1}^{k} P_{i} f\left(A_{i}\right)-f\left(\sum_{i=1}^{k} P_{i} A_{i}\right) \leq f(m) I+f(M) I-2\left[f\left(\frac{M+m}{2}\right)\right] I .
$$

Proof. From Theorem 3.2, we have

$$
\begin{aligned}
& \sum_{i=1}^{k} P_{i} f\left(A_{i}\right)-f\left(\sum_{i=1}^{k} P_{i} A_{i}\right) \\
& \leq U^{*} \operatorname{diag}\left[\left(\alpha_{1} f(m)+\beta_{1} f(M)-f\left(\alpha_{1} m+\beta_{1} M\right)\right), \ldots,\left(\alpha_{n} f(m)+\beta_{n} f(M)-f\left(\alpha_{n} m+\beta_{n} M\right)\right)\right] U .
\end{aligned}
$$

Indeed,

$$
\begin{aligned}
\alpha_{j} f(m)+\beta_{j} f(M) & -f\left(\alpha_{j} m+\beta_{j} M\right) \\
= & f(m)+f(M)-\left[f\left(\alpha_{j} M+\beta_{j} m\right)+f\left(\alpha_{j} m+\beta_{j} M\right)\right] \\
\leq & f(m)+f(M)-2\left[\frac{1}{2} f\left(\alpha_{j} M+\beta_{j} m\right)+\frac{1}{2} f\left(\alpha_{j} m+\beta_{j} M\right)\right] \\
= & f(m)+f(M)-2\left[f\left(\frac{M+m}{2}\right)\right] .
\end{aligned}
$$

Hence,

$$
\sum_{i=1}^{k} P_{i} f\left(A_{i}\right)-f\left(\sum_{i=1}^{k} P_{i} A_{i}\right) \leq f(m) I+f(M) I-2\left[f\left(\frac{M+m}{2}\right)\right] I
$$

Moreover, we get further conclusions

Theorem 3.4. Let $f, A_{i}(i=1, \ldots, k), P_{i}$ be defined as above, and $f$ is also a differentiable convex function, then we have

$$
\sum_{i=1}^{k} P_{i} f\left(A_{i}\right)-f\left(\sum_{i=1}^{k} P_{i} A_{i}\right) \leq \frac{1}{4}(M-m)\left[f^{\prime}(M)-f^{\prime}(m)\right] I .
$$

Proof. Since $f$ is convex, we have

$$
\begin{aligned}
& f\left(\alpha_{j} m+\beta_{j} M\right) \geq f(m)+\beta_{j}(M-m) f^{\prime}(m) ; \\
& f\left(\alpha_{j} m+\beta_{j} M\right) \geq f(M)+\alpha_{j}(m-M) f^{\prime}(M) .
\end{aligned}
$$

Therefore,

$$
\begin{aligned}
& \alpha_{j} f(m)+\beta_{j} f(M)-f\left(\alpha_{j} m+\beta_{j} M\right) \\
& =\alpha_{j}\left[f(m)-f\left(\alpha_{j} m+\beta_{j} M\right)\right]+\beta_{j}\left[f(M)-f\left(\alpha_{j} m+\beta_{j} M\right)\right] \\
& \leq \alpha_{j}\left[f(m)-f(M)-\beta_{j}(M-m) f^{\prime}(m)\right]+\beta_{j}\left[f(M)-f(m)-\alpha_{j}(m-M) f^{\prime}(M)\right] \\
& \leq \alpha_{j} \beta_{j}(m-M) f^{\prime}(M)+\alpha_{j} \beta_{j}(M-m) f^{\prime}(m) \\
& =\alpha_{j} \beta_{j}(M-m)\left[f^{\prime}(M)-f^{\prime}(m)\right] \\
& \leq \frac{1}{4}(M-m)\left[f^{\prime}(M)-f^{\prime}(m)\right]
\end{aligned}
$$


Hence,

$$
\begin{aligned}
\sum_{i=1}^{k} P_{i} f\left(A_{i}\right)-f\left(\sum_{i=1}^{k} P_{i} A_{i}\right) & \leq \max _{\alpha_{j}}\left[\alpha_{j} f(m)+\beta_{j} f(M)-f\left(\alpha_{j} m+\beta_{j} M\right)\right] I \\
& \leq \frac{1}{4}(M-m)\left[f^{\prime}(M)-f^{\prime}(m)\right] I .
\end{aligned}
$$

Thus, the proof is complete.

\section{Authors' contributions}

WJ carried out Matrix operator theory studies, participated in the conception and design. XL conceived of the study, participated in its design and drafting the manuscript. All authors read and approved the final manuscript.

\section{Competing interests}

The authors declare that they have no competing interests. Xue Lanlan is responsible for all the responsibility of the article appeared.

Received: 21 January 2012 Accepted: 27 June 2012 Published: 27 June 2012

\section{References}

1. Beesack, PR, Pěcarić, JE: On Jensen's inequality for convex functions. J Math Anal Appl. 110, 536-552 (1985). doi:10.1016/0022-247X(85)90315-4

2. Kubo, F, Ando, T: Means of positive linear operators. Math Ann. 246, 205-224 (1980). doi:10.1007/BF01371042

3. Hansen, F: An operator inequality. Math Ann. 246, 249-250 (1980). doi:10.1007/BF01371046

4. Gohberg, I, Lancaster, P, Rodman, L: Matrices and Indefinite Scalar Products, OT8. Birkhäuser, Basel (1983)

5. Mond, B, Pećarić, JE: Matrix Inequalities for convex functions. J Math Anal Appl. 209, 147-153 (1997). doi:10.1006/ jmaa.1997.5353

6. Hansen, F: Operator inequalities associated with Jensen's inequality. In: Russia TM (ed.) Survey on Classical Inequalities. pp. 67-98. Kluwer, Dordrecht (2000)

7. Liu, S, Neudecker, H: Several matrix Kantorovich-type inequalities. J Math Anal Appl. 197, $23-26$ (1996). doi:10.1006/ jmaa.1996.0003

8. Simić, S: On a global upper bound for Jensen's inequality. J Math Anal Appl. 343, 414-419 (2008). doi:10.1016/j. jmaa.2008.01.060

doi:10.1186/1029-242X-2012-148

Cite this article as: Lanlan and Junliang: Matrix operator inequalities based on Mond, Pecaric, Jensen and Ando's. Journal of Inequalities and Applications 2012 2012:148.

\section{Submit your manuscript to a SpringerOpen ${ }^{\circ}$ journal and benefit from:}

- Convenient online submission

- Rigorous peer review

- Immediate publication on acceptance

- Open access: articles freely available online

- High visibility within the field

- Retaining the copyright to your article

Submit your next manuscript at $\boldsymbol{\nabla}$ springeropen.com 